\title{
Observations sur l'utilisation de traitements gonadotropes répétés chez la chèvre laitière
}

\author{
G Baril 1, B Rémy 2, JC Vallet 1, JF Beckers 2 \\ 1 INRA, physiologie de la reproduction, 37380 Nouzilly, France; \\ 2 Faculté de Médecine Vétérinaire, 1070 Bruxelles, Belgique
}

(Reçu le 31 mai 1991; accepté le 20 juillet 1991)

\begin{abstract}
Résumé - Trente huit chèvres Saânen ont reçu 2 fois au cours de la même période un traitement gonadotrope de maitrise des cycles (FGA-PMSG). Le pourcentage de femelles venues en cestrus après le second traitement est inférieur à celui observé après le premier traitement $(44,7$ vs $71,0 \%$; $P<0,05$ ). Avant le premier traitement, le pourcentage de liaison de PMSG (\% de PMSG radioactive liée par $10 \mu \mathrm{l}$ de plasma) est plus élevé chez les multipares que chez les nullipares et primipares $(19,2 \pm 22,8$ vs $-0,06 \pm 0,7$ et $1,2 \pm 1,9 ; P<0,01)$, et il augmente après traitement pour toutes les parités. Chez les chèvres traitées 2 fois, le taux de liaison de PMSG avant le second traitement est supérieur à celui observé avant le premier traitement $(22,8 \pm 23,1$ vs $11,2 \pm 19,7 ; P<0,05)$. Quand chez les multipares, le pourcentage de liaison de PMSG avant traitement est faible $(<5 \%)$, les taux de venues en œestrus et de mises-bas après premier traitement ne diffèrent pas de ceux observés chez les nullipares et primipares: la diminution de l'efficacité des traitements ne serait donc pas due à l'âge des animaux. L'utilisation répétée de PMSG durant la vie de la chèvre ou au cours d'une même année est suivie d'une augmentation du niveau d'anticorps anti-PMSG. La présence de ces anticorps expliquerait la diminution de l'efficacité des traitements d'induction et de synchronisation des chaleurs nécessitant l'utilisation de PMSG.
\end{abstract}

PMSG / anticorps / œstrus / ovulation / chèvre

Summary - Observations on the repeated use of gonadotropin treatments in the dairy goat. Our studies have attempted to determine if the absence of oestrus observed in some goats after fluorogestone acetate-pregnant mare serum gonadotropin (FGA-PMSG) treatments could be due to its repeated use. An experiment was carried out in a Saanen flock, in which she-goats were submitted to FGA-PMSG treatment each year. The first treatment of the year was performed on 169 shegoats between March and August 1989. Of the females diagnosed as non-pregnant following the first treatment, 38 were treated a second time during the same period. The proportion of females which went into oestrus after the first treatment was lower in 59 multiparous goats than in 64 nulliparous and in 46 primiparous goats (64.4 vs 100 and $97.8 \% ; \mathrm{P}<0.01$ ). For the she-goats treated twice, the percentage of females which went into oestrus after the second treatment was lower than that observed after the first treatment (44.7 vs $71.0 \% ; \mathrm{P}<0.05)$. The presence of anti$P M S G$ antibodies in plasma was investigated in blood samples taken before and after each treatment. Results are expressed as \% of radioactive PMSG bound by $10 \mu$ l of blood plasma. Before the first treatment, the percentage of bound PMSG was higher in multiparous than in nulliparous and pri- 
miparous goats $(17.5 \pm 23.1$ vs $-0.06 \pm 0.7 ; 1.2 \pm 1.9 ; \mathrm{P}<0.01)$, and increased after treatment for all parities. In goats treated twice, the percentage of bound PMSG before the second treatment was higher than that observed before first treatment $(22.8 \pm 23.1$ vs $11.2 \pm 19.7 ; \mathrm{P}<0.05)$. For both treatments, females which did not come into oestrus showed a percentage of bound PMSG which was significantly higher than that of goats in which oestrus was observed (multiparous first treatment: $30.5 \pm 23.6$ vs $12.9 \pm 20.1 \%$; she-goats in second treatment: $33.9 \pm 23.1$ vs $9.2 \pm 14.5 \%$ ). When the percentage of bound PMSG before treatment was low (<5\%) in multiparous goats, the oestrus and kidding rates after the first treatment did not differ from those observed in nulliparous and primiparous goats. The decrease in the efficiency of the treatments was therefore not due to the age of animals. The repeated use of FGA-PMSG during the lifetime of goats or within the same year is followed by an increase in the levels of antibodies against PMSG. The presence of those antibodies may explain the decrease in the efficiency of these treatments in inducing and synchronizing oestrus.

PMSG / antibodies / oestrus / ovulation / goat

\section{INTRODUCTION}

Chez les caprins de race laitière, l'induction hormonale des chaleurs est utilisée pour déplacer la période de reproduction à des fins économiques; elle est, ou non, associée à l'insémination artificielle. Cette technique dont l'efficacité a été démontrée depuis plusieurs années, connaît en France un développement important (Corteel, 1985). On peut estimer à 150000 le nombre de traitements employés pour les caprins en France en 1988 utilisant des éponges vaginales imprégnées d'acétate de fluorogestone (FGA) et l'injection d'hormone de sérum de jument gravide (PMSG).

À la suite de l'utilisation du traitement FGA-PMSG, plusieurs éleveurs ont cependant constaté l'absence d'œstrus chez certaines chèvres. Par ailleurs, lors de précédents travaux réalisés pour préciser les possibilités de production d'embryons chez les caprins, nous avons observé une diminution du nombre d'ovulations par chèvre donneuse, consécutive à l'utilisation répétée de FSH porcine pour induire la superovulation (Baril et al, 1988). Cette diminution est associée à l'accroissement des niveaux d'anticorps dirigés contre cette hormone non spécifique (Rémy et al, 1991).

Afin de déterminer si un phénomène similaire existait après traitement PMSG, une étude a été réalisée dans un troupeau de race Saânen dont les chèvres sont mises à la reproduction chaque année après traitement FGA-PMSG.

\section{MATÉRIEL ET MÉTHODES}

\section{Induction et synchronisation des chaleurs}

Le premier traitement a été mis en place chez 169 chèvres (64 nullipares, 46 primipares et 59 multipares) entre les mois de mars et août. Durant cette période, 38 femelles diagnostiquées non gestantes après le 1er traitement ont été traitées une seconde fois. L'induction et la synchronisation des chaleurs ont été effectuées selon la méthode utilisée en routine en France (Corteel et al, 1984). Une éponge vaginale imprégnée de 40-45 mg de FGA a été mise en place pour une durée de $11 \mathrm{j} ; 48 \mathrm{~h}$ avant son retrait, chaque femelle a reçu $100 \mu \mathrm{g}$ d'un analogue de prostaglandine $\mathrm{F} 2 \alpha$ (cloprosténol) et 250-600 UI de PMSG selon la période de traitement, la parité et la production laitière des femelles. 


\section{Méthodes de fécondation}

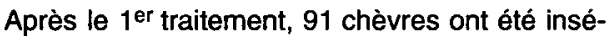
minées, en suivant les conditions requises pour I'IA, 44-46 $\mathrm{h}$ après le retrait de l'éponge avec $200.10^{6}$ spermatozoìdes congelés. Pour 78 et 38 chèvres respectivement après $1^{\text {er }}$ et $2^{\theta}$ traitements, la monte en main a eu lieu au moment de l'observation du début de l'œstrus et 12-18 h plus tard.

La détection de l'œstrus a été effectuée à 24 , 30,36 et $48 \mathrm{~h}$ après le retrait de l'éponge.

\section{Recherche de la présence d'anticorps}

La présence d'anticorps anti-PMSG a été recherchée à partir d'échantillons de sang prélevés avant traitement (à la pose de l'éponge vaginale) et après traitement (au moment du diagnostic de gestation soit 25 j après PMSG). Un test radio-immunologique (Baril et al, 1992) a permis de déterminer le pourcentage de PMSG radio-active liée par $10 \mu \mathrm{l}$ de plasma.

\section{RÉSULTATS ET DISCUSSION}

Suite au 1 er traitement, $87 \%$ des chèvres traitées ont eu un œestrus et $55.6 \%$ ont mis-bas $(49,5 \%$ après $I A$ et $62,8 \%$ après saillie) (tableau I). Chez les multipares, les pourcentages de femelles venues en chaleurs et ayant mis-bas après IA ou saillie sont significativement plus faibles que chez les nullipares et primipares. Pour ces femelles, chez lesquelles l'efficacité du traitement est faible, le taux moyen de liaison de PMSG avant traitement est significativement plus élevé que chez les nullipares et primipares (tableau I). Quelle que soit la parité des femelles, le pourcentage de liaison de PMSG augmente après traitement (tableau II).

Chez les chèvres traitées 2 fois de mars à août, la proportion de femelles venues en chaleurs en $2^{e}$ traitement est si-

Tableau I. Pourcentages de femelles venues en chaleurs et ayant mis-bas suite au fer traitement (de 1989) en relation avec la parité et le taux de liaison de PMSG avant traitement.

\begin{tabular}{|c|c|c|c|c|}
\hline Parité & $\begin{array}{c}\text { Oestrus/ } \\
\text { ch traitées } \\
\text { (\%) }\end{array}$ & $\begin{array}{r}M i \\
\text { après } I A\end{array}$ & $\begin{array}{l}\text { ses bas } \\
\text { après saillie (\%) }\end{array}$ & $\begin{array}{c}\text { Liaison } \\
\text { de PMSG avant } \\
\text { traitement }{ }^{a}(\%)\end{array}$ \\
\hline $\begin{array}{l}\text { Nullipares } \\
(64)\end{array}$ & $\begin{array}{l}100 \\
\text { NS }\end{array}$ & $\begin{array}{l}62,9 \\
(35) \\
\text { NS }\end{array}$ & $\begin{array}{r}79,3 \\
\quad(29) \\
\quad \quad N S\end{array}$ & $\begin{array}{c}-0,06 \pm 0,7 \\
* *\end{array}$ \\
\hline $\begin{array}{l}\text { Primipares } \\
\text { (46) }\end{array}$ & $\begin{array}{c}97,8 \\
\star *\end{array}$ & $\begin{array}{l}51,7 \\
(29) \\
* *\end{array}$ & $\begin{array}{c}82,4 \\
(17) \\
\star \star\end{array}$ & $\begin{array}{c}1,2 \pm 1,9 \\
\star \star\end{array}$ \\
\hline $\begin{array}{l}\text { Multipares } \\
\text { (59) }\end{array}$ & 64,4 & $\begin{array}{l}29,6 \\
(27)\end{array}$ & $\begin{array}{l}37,5 \\
(32)\end{array}$ & $19,2 \pm 22,8$ \\
\hline $\begin{array}{l}\text { Toutes parités } \\
\text { (169) }\end{array}$ & 87 & $\begin{array}{l}49,5 \\
(91)\end{array}$ & $\begin{array}{r}62,8 \\
55.6 \quad(78) \\
\quad\end{array}$ & \\
\hline
\end{tabular}

( ) : n. chèvres; ${ }^{a} m \pm$ écart type; NS : non significatif; ${ }^{\star \star} P<0,01$. 
Tableau II. Pourcentages ( $m \pm$ écart type) de liaison de PMSG avant et après traitement en relation avec la parité des femelles.

Liaison de PMSG (\%)

Parité

\begin{tabular}{ccc}
\hline Nullipares & Primipares & Multipares \\
$-0,06 \pm 0,7$ & $1,2 \pm 1,9$ & $17,5 \pm 23,1$ \\
$4,8 \pm 8,1$ & $28,1 \pm 27,8$ & $42,5 \pm 25,2$ \\
$(64)$ & $(46)$ & $(49)^{a}$
\end{tabular}

a pour 10 femelles non venues en chaleurs les prélèvements de sang après traitement n'ont pas été réalisés. ${ }^{\cdots} P<$ 0,01 .

Tableau III. Pourcentages de chèvres venues en œstrus après 2 traitements successifs en relation avec le taux de liaison de PMSG a.

\begin{tabular}{lccc}
\hline No trait & $\begin{array}{c}\text { Nb } \\
\text { chèvres }\end{array}$ & $\begin{array}{c}\text { Nb oestrus/ } \\
\text { Nb chèvres } \\
\text { traitées (\%) }\end{array}$ & $\begin{array}{c}\text { Liaison } \\
\text { de PMSG } \\
\text { avant traitement } \\
(\%)\end{array}$ \\
\hline $1^{\text {er }}$ & 38 & 71,0 & $11,2 \pm 19,7 \mathrm{~b}$ \\
$2^{\mathrm{e}}$ & 38 & 44,7 & $22,8 \pm 23,1$ \\
\hline
\end{tabular}

a \% de liaison de PMSG avant traitement; ${ }^{b} m \pm$ écart type; $* P<0,05$.

gnificativement plus faible qu'en 1 er traitement et le taux de liaison de PMSG avant $2^{\mathrm{e}}$ traitement est significativement supérieur (tableau III).

Que ce soit pour le premier ou le second traitement, le taux de liaison de PMSG avant traitement est significativement plus élevé chez les chèvres n'ayant pas présenté d'œstrus que chez celles où il a été observé (multipares 1 er traitement :
$30,5 \pm 23,6$ vs $12,9 \pm 20,1 \%$; chèvres en $2^{e}$ traitement $33,9 \pm 23,1$ vs $9,2 \pm 14,5 \%$ ).

Les femelles nullipares et primipares dont les taux de venue en cestrus sont élevés présentent avant traitement des pourcentages de liaison de PMSG nuls ou faibles, et ceux-ci ne diffèrent pas selon qu'elles ont mis-bas ou non à la suite du $1^{\text {er }}$ traitement (tableau IV). Chez les multipares, le taux de liaison de PMSG avant le $1^{\text {er }}$ traitement est plus élevé pour les femelles n'ayant pas mis bas que pour celles ayant mis bas $(25,7 \pm 23,3$ vs $6,5 \pm 15,9$; tableau IV).

Quand pour les multipares, le pourcentage de liaison de PMSG avant traitement est faible $(<5 \%)$, les taux de venue en œstrus et de mise-bas sont comparables à ceux observés chez les nullipares et primipares (tableau V). Par conséquent, la faible efficacité du traitement chez les multipares n'est pas due à l'âge des femelles.

\section{CONCLUSION}

Dans le troupeau où cette étude a été réalisée, la proportion de chèvres n'ayant pas eu d'œstrus est la plus élevée chez les fe- 
Tableau IV. Pourcentages de liaison de PMSG avant $1^{\text {er }}$ traitement de 1989 en relation avec la fertilité après ce traitement.

\begin{tabular}{lccc}
\hline & \multicolumn{3}{c}{ Parité } \\
\cline { 2 - 4 } & Nullipares & Primipares & Multipares \\
\hline Chèvres ayant mis bas après 1er traitement & $\begin{array}{c}-0,05 \pm 0,8 \\
(45)\end{array}$ & $\begin{array}{c}1,2 \pm 1,6 \\
(29)\end{array}$ & $\begin{array}{c}6,5 \pm 15,9 \\
(20)\end{array}$ \\
Chèvres n'ayant pas bas mis bas après 1er traitement & NS & NS & $*, 07 \pm 0,4$ \\
& $(19)$ & $\begin{array}{c}1,2 \pm 2,3 \\
(17)\end{array}$ & $\begin{array}{c}25,7 \pm 23,3 \\
(39)\end{array}$ \\
\hline
\end{tabular}

() : $n$ de chèvres; a $m \pm$ écart type; NS : différence non significative; ** $P<0,01$.

Tableau V. Effet du taux de liaison de PMSG avant le 1er traitement de 1989 sur les pourcentages de chèvres multipares venues en œstrus et ayant mis bas.

\begin{tabular}{|c|c|c|c|c|}
\hline \multirow{2}{*}{$\begin{array}{l}\text { Pourcentage } \\
\text { de liaison } \\
\text { avant traitement }\end{array}$} & \multirow{2}{*}{$\begin{array}{c}\text { Oestrus/ } \\
\text { ch traitées } \\
(\%)\end{array}$} & \multicolumn{3}{|c|}{ Mise bas (\%) } \\
\hline & & monte en main & $I A$ & monte en main et $I A$ \\
\hline$\leq 5.0$ & $\begin{array}{l}95,8 \\
(24) \\
* \star\end{array}$ & $\begin{array}{l}69,2 \\
(13) \\
\star \star \star\end{array}$ & $\begin{array}{l}63,6 \\
(11) \\
* *\end{array}$ & $\begin{array}{l}66,7 \\
(24) \\
* \star\end{array}$ \\
\hline$>5.0$ & $\begin{array}{l}42,9 \\
(35)\end{array}$ & $\begin{array}{l}15,8 \\
(19)\end{array}$ & $\begin{array}{r}6,3 \\
(16)\end{array}$ & $\begin{array}{l}11,4 \\
(35)\end{array}$ \\
\hline Total & $\begin{array}{l}64,4 \\
(59)\end{array}$ & $\begin{array}{l}37,5 \\
(32)\end{array}$ & $\begin{array}{l}29,6 \\
(27)\end{array}$ & $\begin{array}{l}33,9 \\
(59)\end{array}$ \\
\hline
\end{tabular}

( ) : n chèvres; "* $P<0,01$.

melles multipares déjà traitées au moins 2 fois au cours de leur vie, et chez les chèvres soumises à second traitement au cours de la même année. Chez ces femelles, la présence d'anticorps révélée par les hauts niveaux de liaison de PMSG est consécutive à l'utilisation répétée de ce traitement et conduit à une diminution de son efficacité.

Afin de vérifier l'importance de ce phénomène, cette étude est poursuivie dans d'autres troupeaux. Dans l'immédiat, il est souhaitable d'éviter de traiter les chèvres plus d'une fois par an. 


\section{RÉFÉRENCES}

Baril G, Casamitjana P, Perrin J, Vallet JC (1988) Embryo production, freezing and transfer in Angora, Alpine and Saanen goats. European embryo transfer Assoc 4th Scientific Meeting. 9-10 Septembre 1988, Lyon, France, 67-93

Baril G, Remy B, Vallet JC, Beckers JF (1992) Effect of repeated use of progestagen-PMSG treatment for estrus control in dairy goats out of breeding season. Zuchthygiene (accepté pour publication)

Corteel JM (1985) Maîtrise de la reproduction des caprins à vocation laitière à des fins économiques. In: $1^{\text {er }}$ colloque int reprod caprins.
26 Avril 1985, Drummondville, Québec, Canada, $45-68$

Corteel JM, Nunes JF, Dahuron C, Gonzales CS, Baril G, Leboeuf $B$, Boué P, Loysel C, de Montigny $G$ (1984) La congélation du sperme et l'induction hormonale de l'œstrus et de l'ovulation chez les caprins à vocation laitière. $C R 9^{\circ}$ Joum rech ovine caprine. 5 et 6 Décembre 1984, Paris, France 152-172

Remy B, Baril G, Vallet JC, Dufour R, Chouvet $C$, Saumande J, Chupin D, Beckers J F (1991) Are antibodies responsible for a decreased superovulatory response in goats which have been treated repeatedly with porcine follicle-stimulating-hormone? Theriogenology $36,389-399$ 